\title{
Few-electron eigenstates of concentric double quantum rings
}

\author{
B. Szafran ${ }^{1,2}$ and F. M. Peeters ${ }^{1, *}$ \\ ${ }^{1}$ Departement Fysica, Universiteit Antwerpen, Groenenborgerlaan 171, B-2020 Antwerpen, Belgium \\ ${ }^{2}$ Faculty of Physics and Applied Computer Science, AGH University of Science and Technology, al. Mickiewicza 30, \\ 30-059 Kraków, Poland
}

(Received 27 June 2005; revised manuscript received 26 August 2005; published 19 October 2005)

\begin{abstract}
Few-electron eigenstates confined in coupled concentric double quantum rings are studied by the exact diagonalization technique. We show that the magnetic field suppresses the tunnel coupling between the rings, localizing the single-electron states in the internal ring and the few-electron states in the external ring. The magnetic fields inducing the ground-state angular momentum transitions are determined by the distribution of the electron charge between the rings. The charge redistribution is translated into modifications of the fractional Aharonov-Bohm period. We demonstrate that the electron distribution can be deduced from the cusp pattern of the chemical potentials governing the single-electron charging properties of the system. The evolution of the electron-electron correlations to the high field limit of a classical Wigner molecule is discussed.
\end{abstract}

DOI: $10.1103 /$ PhysRevB.72.155316

PACS number(s): 73.21.La

\section{INTRODUCTION}

The phase shift of the electron wave function by the vector potential ${ }^{1}$ results in oscillations of the quantum transport properties $^{2-6}$ of ring-shaped structures. The conductance ${ }^{7}$ of metal and semiconductor rings is periodic in the external magnetic field with a period determined by the magnetic flux through the ring. On the other hand, in bound states of closed circular quantum rings, the single-electron spectrum exhibits periodic ground-state angular momentum transitions with the period of the flux quantum. ${ }^{8}$ In confined interacting fewelectron systems fractional Aharonov-Bohm (AB) periodicity of the spectrum was predicted ${ }^{9,10}$ and subsequently observed in conductance oscillations measured ${ }^{11}$ in a transport spectroscopy experiment. Discussion of the fractional periodicity in the context of the strength of the electron-electron interaction was given in Ref. 12. The fractional period for the interacting electron system is also found in realistic modeling of InGaAs self-assembled quantum rings. ${ }^{13}$

Recently, fabrication of self-assembled strain-free double concentric GaAs/AlGaAs quantum rings was reported. ${ }^{14}$ Concentric coupled quantum ring structures can also be produced by the atomic force microscope tip oxidation technique. ${ }^{4,11}$ In this paper we present an exact diagonalization study of the properties of few-electron states confined in concentric quantum rings. In the presence of inter-ring tunnel coupling the electron wave functions undergo hybridization, forming molecular orbitals similarly as in artificial molecules formed by lateral ${ }^{15-19}$ or vertical ${ }^{20-22}$ coupling of quantum dots. The magnetic field $A B$ period will be significantly different for the internal and external rings. Therefore, the question arises, what will be the periodicity of the angular momentum transitions for such hybridized orbitals?

In the two-electron laterally coupled dots, the external magnetic field enhances the localization of the wave functions in each of the dots. ${ }^{17}$ Similar is the effect of the electron-electron interaction favoring charge segregation. On the other hand, in concentric rings the electron-electron interaction will favor localization of the electrons in the external ring while the diamagnetic term of the Hamiltonian will tend to localize the electrons in the inner ring. We will show that the redistribution of the electrons between the rings affects the $\mathrm{AB}$ period of the angular momentum transitions, which can be extracted from conductance measurements ${ }^{11}$ on rings connected to electrodes. Moreover, the angular momentum transitions result in characteristic cusp patterns of the chemical potential determining the single-electron charging of the structure. The alignment of the chemical potentials of the confined electrons with the Fermi level of the gate electrode can be detected in capacitance spectroscopy, which was used earlier to study the electronic structure of selfassembled quantum rings ${ }^{23}$ incorporated in a charge tunable structure.

The present paper extends our previous work on the coupling between a quantum dot and a quantum ring. ${ }^{24}$ For a single quantum ring, the envelope of the single-electron ground-state energy depends only on the strength of the confinement in the radial direction and not on the radius of the ring. For the radial ring confinement energy $\hbar \omega$, when the radius of the ring is large as compared to the range of the radial confinement, the ground-state envelope is approximately given ${ }^{24}$ by $\sqrt{(\hbar \omega)^{2}+\left(\hbar \omega_{c}\right)^{2}} / 2$, where $\omega_{c}$ is the cyclotron frequency. Therefore, a continuous evolution of the electron distribution between the two rings should be expected as a function of the magnetic field in contrast to the rapid ground-state charge redistributions found previously for a quantum dot coupled to a surrounding quantum ring. ${ }^{24}$

A study related to the present one was presented earlier for two concentric superconducting rings ${ }^{25}$ in which the coupling between the rings was mediated by the magnetic selffield of the separate rings.

The paper is organized as follows. In Sec. II we present the model, the results for the single-electron coupling are given in Sec. III, and for the interacting electron systems in Sec. IV. Section V contains the summary and conclusions.

\section{THEORY}

We consider a two-dimensional model of circularly symmetric double concentric rings with confinement potential taken in the form 


$$
V(\rho)=\frac{m \omega^{2}}{2} \min \left[\left(\rho-R_{1}\right)^{2},\left(\rho-R_{2}\right)^{2}\right],
$$

where $m$ is the effective electron band mass, $R_{1}$ and $R_{2}$ stand for the internal and external ring radii, $\rho$ is the distance of the origin, and $\omega$ is the harmonic oscillator frequency for the lateral confinement of the electrons in each of the rings. Similar models were previously applied for laterally coupled dots. ${ }^{17-19}$ In our calculations we take the GaAs value for the mass $m=0.067 m_{0}$, the dielectric constant $\epsilon=12.4$, and assume $\hbar \omega=3 \mathrm{meV}$. The adopted oscillator energy corresponds to a length $l=\sqrt{2 \hbar / m \omega}=27.5 \mathrm{~nm}$ which defines the width $d=2 l$ of the considered rings. The Hamiltonian of a single electron in a perpendicular magnetic field $(B)$, using the symmetric gauge, is

$$
h=-\frac{\hbar^{2}}{2 m}\left(\frac{d^{2}}{d \rho^{2}}+\frac{1}{\rho} \frac{d}{d \rho}\right)+\frac{\hbar^{2} L^{2}}{2 m \rho^{2}}+\frac{m \omega_{c}^{2} \rho^{2}}{8}-\frac{1}{2} \hbar \omega_{c} L+V(\rho),
$$

where $L$ is the angular momentum of the considered state, and $\omega_{c}=e B / \mathrm{m}$. In the following we refer to the second, third, and fourth term of the Hamiltonian as the centrifugal, diamagnetic, and the orbital Zeeman terms. We neglect the Zeeman interaction of the electron spin with the magnetic field, which at high fields polarizes the spins of the confined electrons. The spin Zeeman interaction is decoupled from the orbital degree of freedom, it does not influence the tunnel coupling, and can be trivially accounted for as an energy shift linear in $B .{ }^{24}$ The eigenstates of the $N$-electron Hamiltonian

$$
H=\sum_{i=1}^{N} h_{i}+\sum_{i=1}^{N} \sum_{j>i}^{N} \frac{e^{2}}{4 \pi \epsilon \epsilon_{0} r_{i j}}
$$

are found with a standard ${ }^{26,27}$ exact diagonalization approach using the single-electron eigenstates of operator (2) to construct the basis elements in the form of Slater determinants. We use the numerical method as originally developed to discuss the coupling between a quantum dot and a quantum ring. ${ }^{24}$ The single-electron Hamiltonian (2) is diagonalized using a finite difference scheme and the Coulomb matrix elements are integrated numerically.

\section{SINGLE ELECTRON COUPLING}

Let us first discuss the single-electron states in the coupled concentric rings. Figure 1 shows the potential felt by an electron in the $L=0$ and $L=6$ states as well as the lowestenergy orbitals (radial probability densities) for $R_{1}=120 \mathrm{~nm}$ and $R_{2}=180 \mathrm{~nm}$ in the absence of a magnetic field. In the lowest $L=0$ states the electron is equally probable to be found in both rings and the orbitals possess a clear bonding and antibonding character. On the other hand, for $L \neq 0$, the centrifugal potential pushes the electrons towards the outer ring. In Fig. 1 we show the result for $L=6$, which clearly shows that the lowest-energy orbital is shifted to the external ring. As a consequence, the electron in the excited-state orbital occupies predominantly the inner ring and the zero of

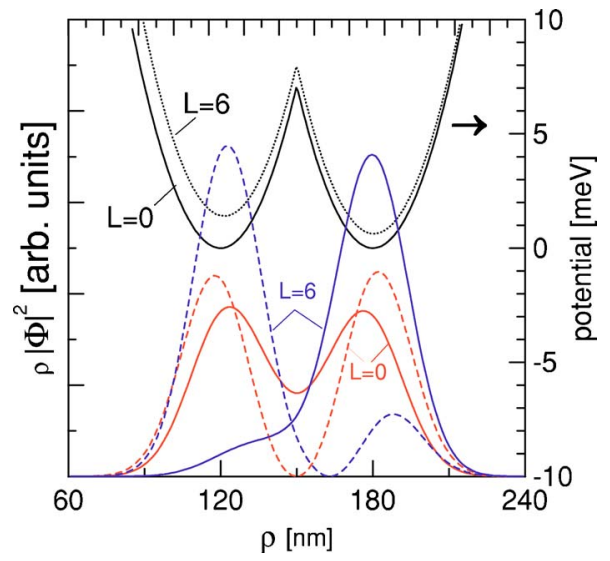

FIG. 1. (Color online) Radial profile of the confinement potential (black solid curve referred to the right vertical axis) of the two concentric rings for $R_{1}=120 \mathrm{~nm}$ and $R_{2}=180 \mathrm{~nm}$ at $B=0$. The black dotted curve shows the sum of the confinement potential and the centrifugal potential for $L=6$. Red (light gray) and blue (dark gray) curves show the square of the modulus of the two lowest-energy single-electron wave functions multiplied by Jacobian $\rho$ at $B=0$ for $L=0$ and $L=6$, respectively. The lower-energy orbitals are given by the solid curves and the higher-energy orbitals by the dashed curves.

the wave function is displaced from the center of the barrier to the external ring. We see that the bonding-antibonding character of the lowest-energy orbitals occupying both rings is, for increasing $L$, replaced by a single-ring type of localization. Therefore, the effect of the centrifugal potential is to lift the tunnel coupling.

The energy levels are shown in Fig. 2 as functions of the inner ring radius $R_{1}$ for fixed $R_{2}=180 \mathrm{~nm}$. Note that for $R_{1}$ $=0$, the system consists of a quantum dot surrounded by a quantum ring. ${ }^{24}$ The lowest-energy level for $L=0$ and $B=0$ [see Fig. 2(a)] is then associated with the ring-localized state (of energy close to $\hbar \omega / 2=1.5 \mathrm{meV}$ ) and the excited state corresponds to an electron confined in the parabolic quantum dot (of energy $\hbar \omega=3 \mathrm{meV}$ ). For $R_{1}>0$ the quantum dot is transformed to a quantum ring. The energy of the orbital, which is predominantly localized in the inner ring, first goes below $\hbar \omega / 2$ and then returns to this value. Around $R_{1}$ $=80 \mathrm{~nm}$ the tunnel coupling appears between the internal and the external rings, leading to an energy gap between the two energy levels. Finally, for a single quantum ring $\left(R_{1}=R_{2}\right.$ $=180 \mathrm{~nm})$ the spectrum resembles the one-dimensional harmonic oscillator potential. ${ }^{24}$ For $L=2$ at $R_{1}=0$ both the lowest-energy levels correspond to orbitals localized in the external ring. The energies are slightly shifted above $\hbar \omega / 2$ and $3 \hbar \omega / 2$ by the centrifugal potential. The internal ring localized level becomes the first excited state near $R_{1}$ $=30 \mathrm{~nm}$. The centrifugal potential lowers the height of the inter-ring tunnel barrier (see Fig. 1). Consequently, the avoided crossings between the $L=2$ energy levels $\left(R_{1}\right.$ $\simeq 100 \mathrm{~nm}$ ) are visibly larger than for $L=0$. A larger centrifugal shift of the energy levels and a stronger level interaction, a signature of a stronger tunnel coupling, is observed for $L$ $=6$ [see Fig. 2(b)]. For $L=6$ and $B=0.5 \mathrm{~T}$ the diamagnetic shift of the external ring-confined level is almost exactly 

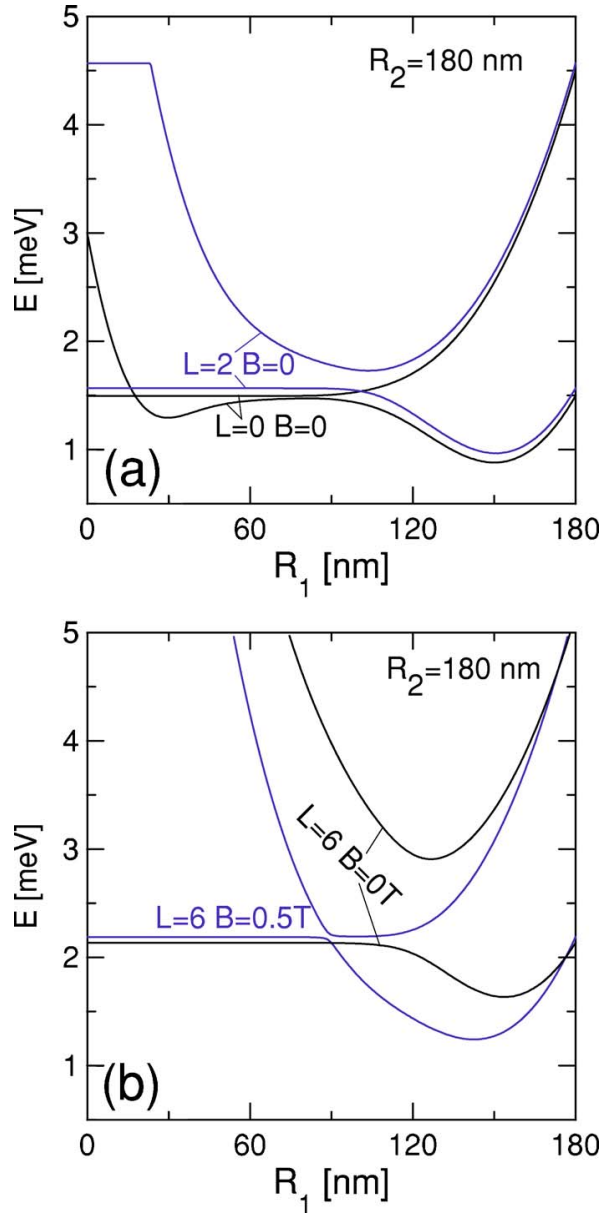

FIG. 2. (Color online) Two lowest single-electron energy levels for $L=0$, and $L=2$ at $B=0$ (a) and for $L=6$ at $B=0$ and $0.5 \mathrm{~T}$ (b), as functions of the internal ring radius for an external ring of radius $R_{2}=180 \mathrm{~nm}$.

cancelled by the orbital Zeeman term [compare the lowest black and blue curves at $R_{1}=0$ in Fig. 2(b)]. However, the Zeeman term dominates for the state localized in the internal ring. As a consequence, the energy levels change their order in a narrow anticrossing near $R_{1}=90 \mathrm{~nm}$.

The dependence of the single-electron energy spectrum on the external magnetic field is plotted in Figs. 3(a)-3(c) for fixed $R_{2}=180 \mathrm{~nm}$ and different internal ring radii. For $R_{1}$ $=60 \mathrm{~nm}$ there is no tunneling between the rings and the spectrum is a simple sum of two single-ring spectra. The spectrum corresponding to the internal ring exhibits angular momentum transitions with a period of $0.214 \mathrm{~T}$ while the period of the one corresponding to the external ring is $0.0406 \mathrm{~T}$. These periods correspond to the flux quantum passing through an effective one-dimensional ring of radius $55.4 \mathrm{~nm}$ and $180 \mathrm{~nm}$, respectively. The ground state corresponds to the electron in the internal ring, except for $B \simeq 0.2 \mathrm{~T}$ and $B$ $\simeq 0.65 \mathrm{~T}$. The inner-ring localized states are favored by the $-(1 / \rho)(d / d \rho)$ term of the kinetic energy.

For $R_{1}=100 \mathrm{~nm}$ [see Fig. 3(b)] the inter-ring coupling is non-negligible. For comparison, the ground-state energy of the single quantum ring of radius $180 \mathrm{~nm}$ is also shown in Fig. 3(b) by the black curve. For $B>0.15 \mathrm{~T}$, sightly above the ground state, we observe more frequent angular momentum transitions than in the ground state. This energy band corresponds to the electron predominantly confined in the external ring. With increasing magnetic field, this band closely approaches the single-ring spectrum (cf. the black curve), which indicates that the electron becomes entirely localized in the external ring. Thus at high magnetic fields the spectrum of the internal and external rings become decoupled. Note that the energy band corresponding to the localization of the electron in the external ring becomes distinct only for $L>4$.

Energy levels with the same angular momentum change their order through avoided crossings. The lowest-energy levels, for $L \geqslant 2$, possess two minima, after and before the avoided level crossing. The wave functions and the potentials for the anticrossing of the $L=5$ energy levels [see the anticrossing of red lines near $0.38 \mathrm{~T}$ at Fig. 3(b)] are presented in Fig. 3(d). The $L=5$ eigenstate for $B=0.2 \mathrm{~T}$ is the lowest-energy state of the external ring energy band [see Fig. 3 (b) and the paragraph above] and its wave function is predominantly localized in the outer ring [see Fig. 3(d)]. At $B$ $=0.38 \mathrm{~T}$, corresponding to the smallest distance between the anticrossing energy levels, the electron can be found with a comparable probability in both rings. After the avoided crossing the diamagnetic potential localizes the electron in the internal ring. For $B=0.7 \mathrm{~T}$ the $L=5$ state is localized almost entirely in the inner ring [see purple curve in Fig. $3(\mathrm{~d})$ ] when it corresponds to the ground-state of the system [Fig. 3(b)]. Concluding, for $B=0$ and fixed nonzero $L$ the lowest energy level is predominantly localized in the external ring due to the centrifugal potential. For high magnetic field, the lowest-energy state for a fixed $L$ is transferred to the internal ring by the diamagnetic term of the Hamiltonian.

For $R_{1}=120 \mathrm{~nm}$ [Fig. 3(c)] the coupling between the two rings is stronger and the difference between the centrifugal potentials in both rings is smaller. Consequently the two decoupled spectra of the internal and external ring are only distinguishable for $B>0.5 \mathrm{~T}$. The amount of electron charge localized in the internal ring [integrated over $\rho$ from 0 to $\left.\left(R_{1}+R_{2}\right) / 2\right]$ for the ground state is plotted in Fig. 4, together with the ground-state angular momentum. For low magnetic field the ground-state wave functions are almost equally distributed between the two rings and at high field they are entirely localized in the inner ring. Consequently, the period of the ground-state oscillations increases with $B$ (see the slope of the black staircase in Fig. 4). Note that the decoupling of the spectra in Fig. 3(c) for $B>0.5 \mathrm{~T}\left(R_{1}=120 \mathrm{~nm}\right)$ is accompanied by the transfer of the electron to the internal ring (see Fig. 4). For $R_{1}=140 \mathrm{~nm}$ many more angular momentum transitions are needed before the electron becomes entirely localized in the inner ring.

At the end of this section we would like to explain the role of the adopted finite value of the rings' width for our results. The studied rings radii $(R \simeq 150 \mathrm{~nm})$ and width $(d$ $=55 \mathrm{~nm})$ correspond to structures produced by the tip oxidation technique. ${ }^{4}$ For instance, the ring of Ref. 4 is characterized by $R=132 \mathrm{~nm}$ and $d=65 \mathrm{~nm}$. In the limit of infinite oscillator energy $(\hbar \omega)$ the rings become strictly onedimensional $(d \rightarrow 0)$ and decoupled due to the infinite inter- 

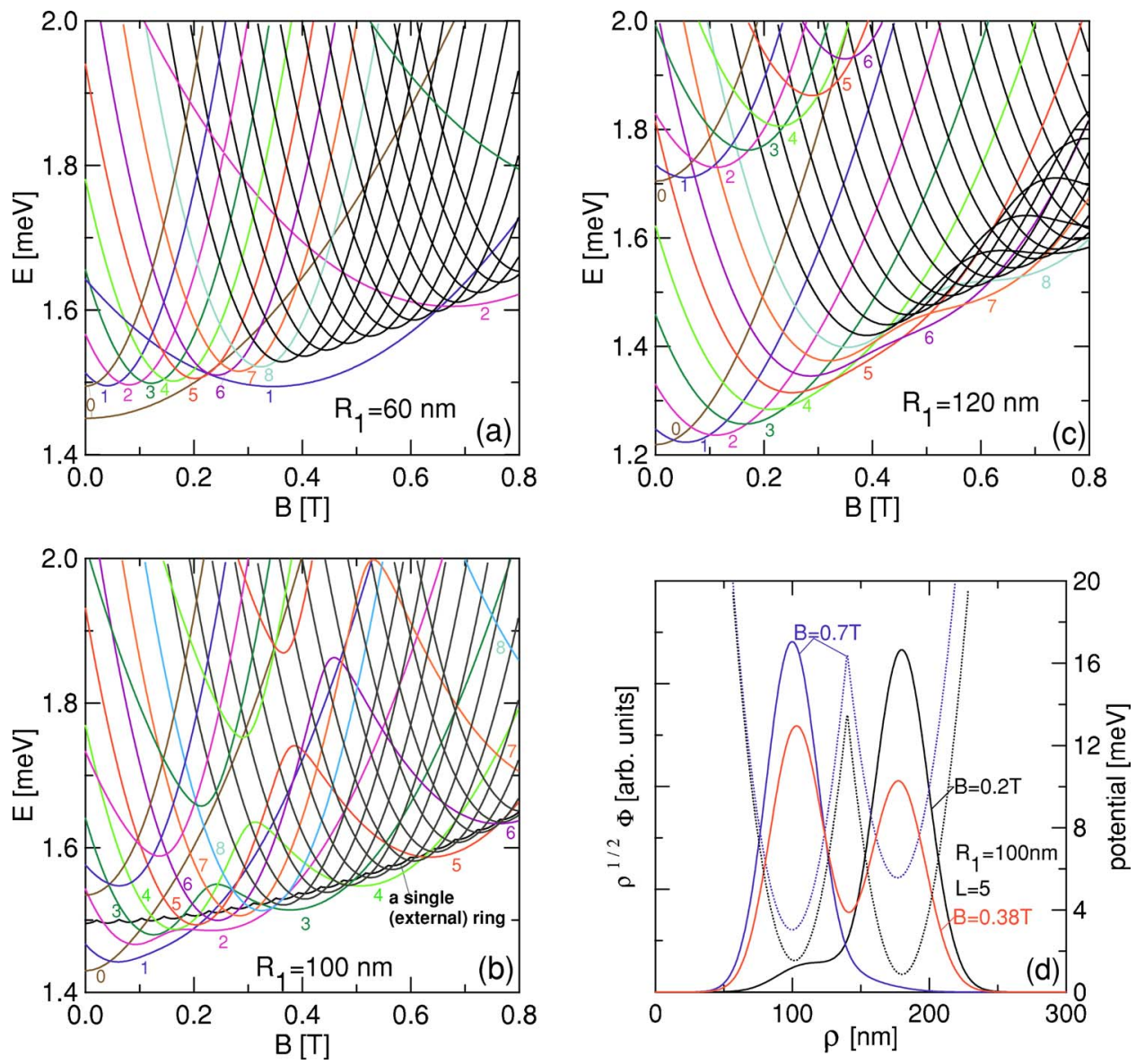

FIG. 3. (Color online) (a)-(c) Single electron spectrum for coupled rings with the external ring radius $R_{2}=180 \mathrm{~nm}$ and the internal ring radius $R_{1}=60 \mathrm{~nm}$ (a), $100 \mathrm{~nm}$ (b), and $120 \mathrm{~nm}$ (c). Energy levels corresponding to different angular momenta up to 8 were plotted with different colors. In (b) the ground state of a single ring with radius $180 \mathrm{~nm}$ is shown by the black curve. (d) Lowest energy $L=5$ wave function (solid lines) for $R_{1}=100 \mathrm{~nm}$, before $(B=0.2 \mathrm{~T})$ at $(0.38 \mathrm{~T})$ and after $(0.7 \mathrm{~T})$ the avoided crossings of the energy levels [cf. red lines in (b) ] corresponding to states localized in the external and internal ring, respectively. Dotted curves refer to the right vertical axis and show the sum of the confinement, centrifugal, and diamagnetic potentials.

ring barrier. The energy levels of states confined in onedimensional rings only depend on the magnetic flux ${ }^{8} E_{i}(L)$ $=\left[\hbar^{2} /\left(2 m R_{i}^{2}\right)\right]\left(L-\Phi_{i} / \Phi_{0}\right)^{2}$, where $i=1,2$ stands for the internal and external ring localization respectively, $\Phi_{0}=h / e$ is the flux quantum, and $\Phi_{i}$ corresponds to the flux through the radius $R_{i}$. It is clear that the localization of the lowest-energy level of a pair of one-dimensional rings will oscillate abruptly between internal and external rings when the magnetic field is increased. However, this switching is deprived of physical consequences since due to the infinite inter-ring barrier the electron is not allowed to release its energy tunneling from one ring to the other. Note that a trace of the discussed localization switching can be observed in Fig. 3(a) for negligible inter-ring tunnel coupling. Decoupled spectra with short appearances of the external ring localization in the lowest-energy state similar to Fig. 3(a) are obtained for $R_{1}$ $=120 \mathrm{~nm}, R_{2}=180 \mathrm{~nm}$ for $d$ decreased from $55 \mathrm{~nm}$ [as in Fig. 3(c)] to $13.5 \mathrm{~nm}(\hbar \omega=50 \mathrm{meV})$. The rapid localization switching disappears for the nontrivial case of a nonnegligible tunnel coupling [cf. Figs. 3(b)-3(d)].

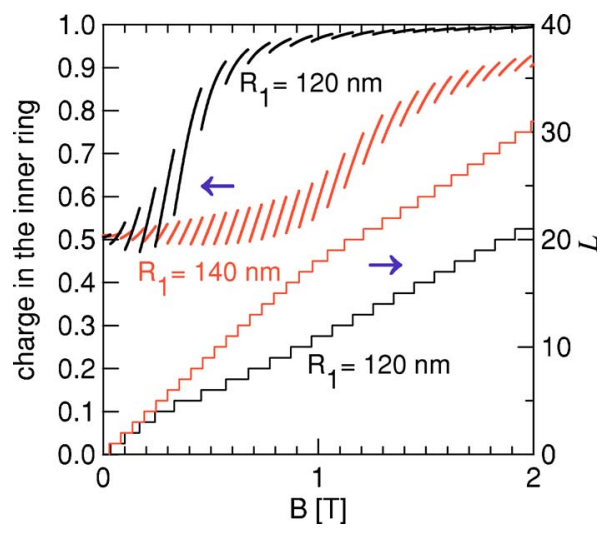

FIG. 4. (Color online) The discontinuous lines show the amount of charge localized in the internal ring for the single-electron ground state. The results correspond to the external radius $R_{2}$ $=180 \mathrm{~nm}$ and internal radius $R_{1}=120 \mathrm{~nm}$ (black lines) and $R_{1}$ $=140 \mathrm{~nm}$ (red lines) as functions of the magnetic field. The staircases at the lower part of the figure are referred to the right axis and show the ground-state angular momentum. 

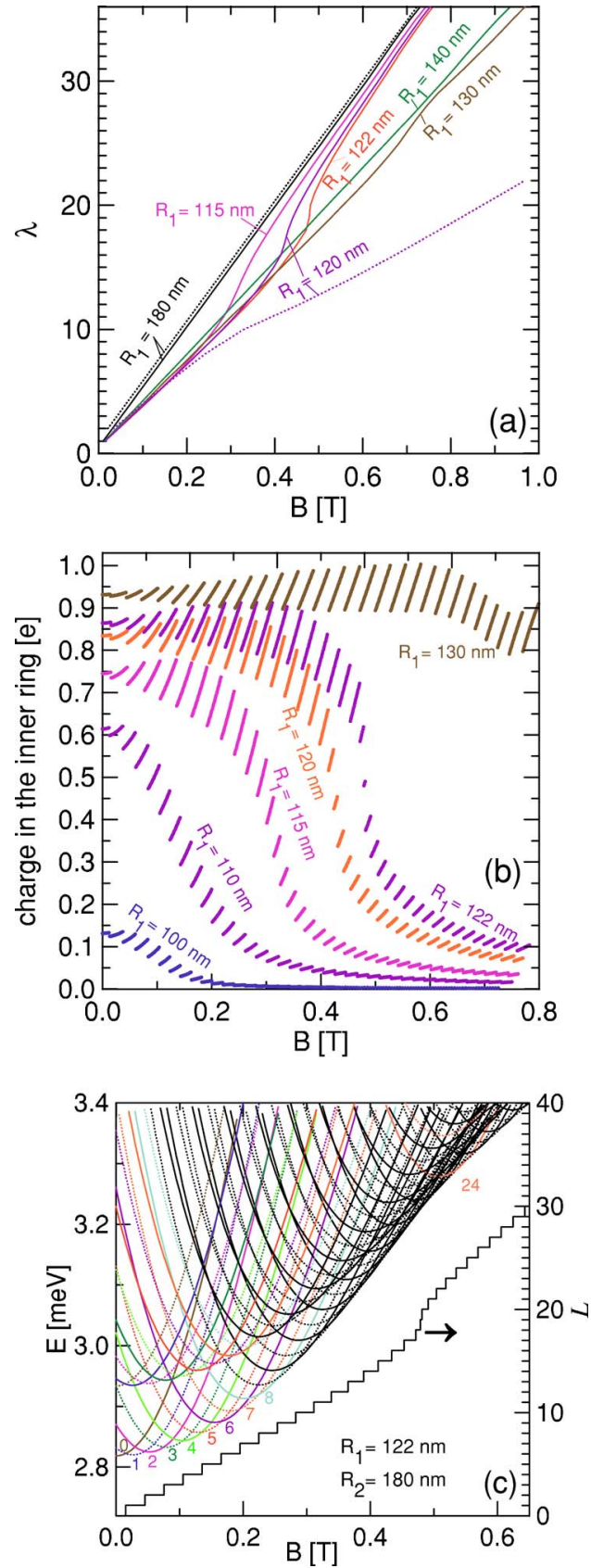

FIG. 5. (Color online) (a) Upper bound for the two-electron ground-state angular momentum for $R_{2}=180 \mathrm{~nm}$ and various values of the inner ring radius. The dotted lines show the values in the absence of the electron-electron interaction. (b) Charge localized in the inner ring as function of the magnetic field for $R_{2}=180 \mathrm{~nm}$ and different radii of the inner ring. (c) The two-electron energy spectrum for $R_{1}=122 \mathrm{~nm}$ and $R_{2}=180 \mathrm{~nm}$. The spin singlets are plotted as solid lines and the triplets with dotted lines. In the bottom of the figure the ground-state angular momentum staircase is plotted.

\section{FEW-ELECTRON EIGENSTATES}

Let us now discuss the effect of the electron-electron interaction on the ground-state properties of few-electron systems. We find that for the interacting two-electron system the ground-state angular momentum takes on all the subsequent integer values, such as for a single quantum ring. The upper
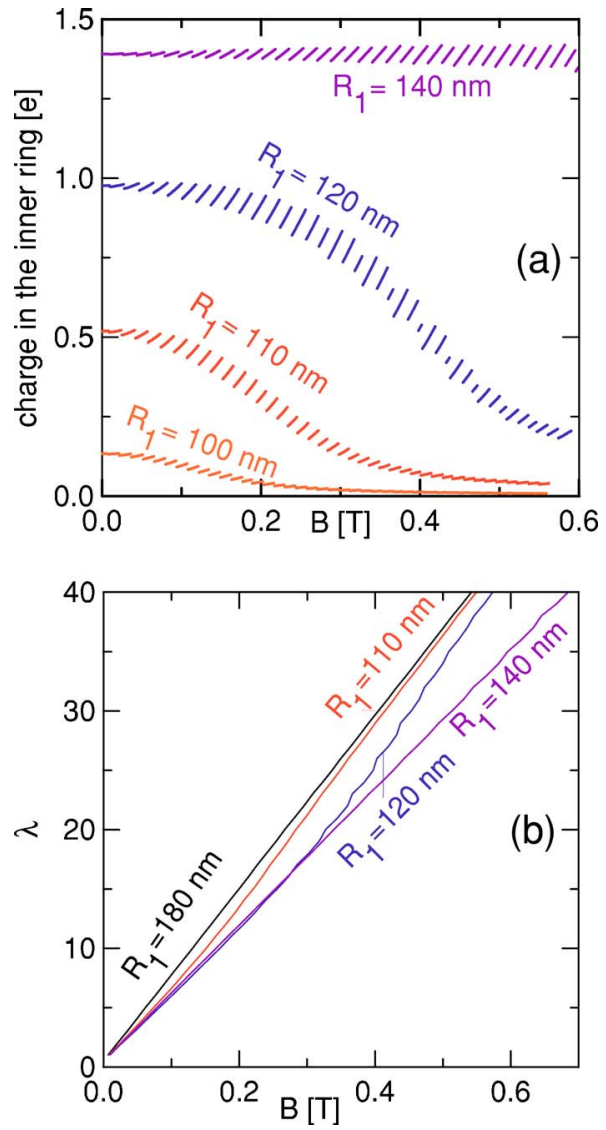

FIG. 6. (Color online) Three electrons in two concentric rings. (a) Charge localized in the inner ring for $R_{2}=180 \mathrm{~nm}$ and various $R_{1}$ radii. (b) Upper bound for the ground-state angular momentum.

bound for the ground-state angular momentum of the twoelectron system $\lambda \geqslant L$ is plotted in Fig. 5(a) as a function of the external magnetic field. In contrast to the single-electron problem, no influence of the inner ring on the ground-state angular momentum is observed for $R_{1}<90 \mathrm{~nm}$. This indicates that the Coulomb repulsion prevents the electrons from occupying the inner ring if its radius is too small. As a signature of the inter-ring coupling we see for $R_{1}=115 \mathrm{~nm}, 120$ $\mathrm{nm}$, and $122 \mathrm{~nm}$ that the ground-state angular momentum increases initially more slowly than for the single $R$ $=180 \mathrm{~nm}$ ring, indicating the presence of electron charge in the internal ring. At a certain value of magnetic field, however, the lines change their slope and tend toward the values obtained for a single ring of radius $180 \mathrm{~nm}$. In the discussed range of the magnetic field, the inter-ring coupling for the internal ring radii $R_{1}=130$ and $140 \mathrm{~nm}$ is preserved.

Dotted lines in Fig. 5(a) show the $\lambda$ values for the noninteracting electron couple for a single ring with $R=180 \mathrm{~nm}$ (black dots) and for the double ring with $R_{1}=120 \mathrm{~nm}$ and $R_{2}=180 \mathrm{~nm}$ (orange dots). For the single $R=180 \mathrm{~nm}$ ring, the $\lambda$ values for the interacting and noninteracting cases run parallel to one another. However, for $R=120 \mathrm{~nm}$ the $\lambda$ values for the noninteracting pair decreases its slope as the magnetic field is increased, while for the interacting pair an increase of the slope is observed instead. This is because for high magnetic fields the interacting electrons tend to occupy the external ring [cf. Fig. 5(b)] to minimize their mutual repulsion 


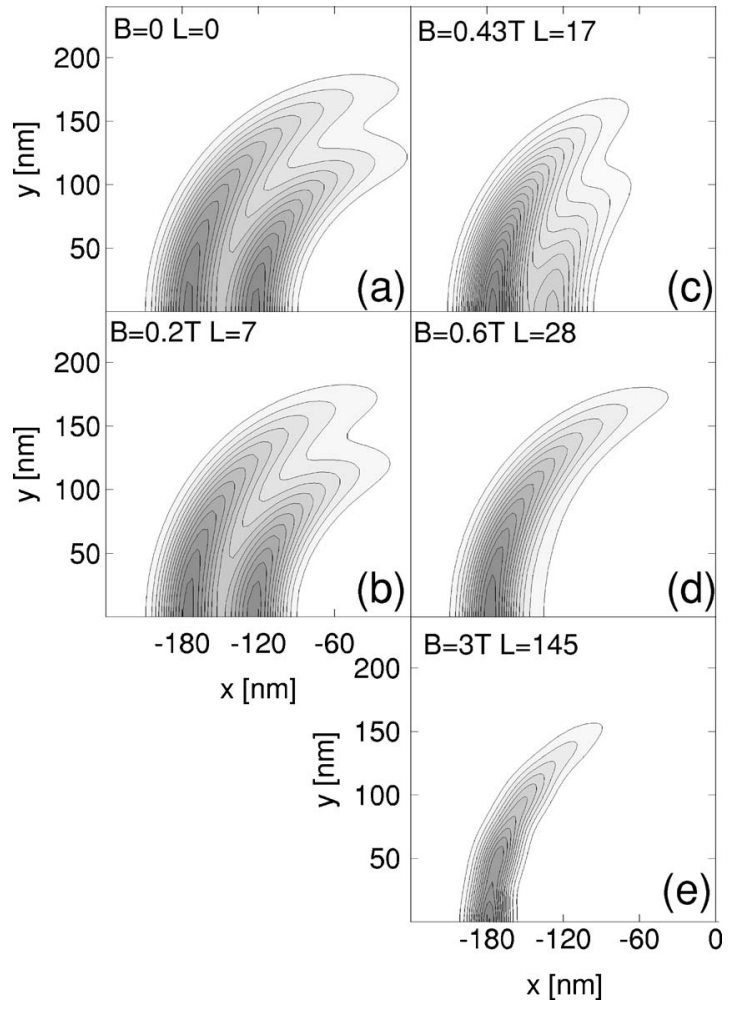

FIG. 7. Pair correlation function for two-electron ground state in concentric rings with radii $R_{1}=120 \mathrm{~nm}$ and $R_{2}=180 \mathrm{~nm}$. One of the electrons is fixed at the point $(180 \mathrm{~nm}, 0)$.

in contrast to the single-electron problem in which the diamagnetic term of the Hamiltonian promotes the localization in the inner ring (see Fig. 4).

The energy spectrum for $R_{1}=122 \mathrm{~nm}$, for which the localization of the charge in the external ring appears in the most abrupt way, is plotted in Fig. 5(c). Below $B=0.4 \mathrm{~T}$ one can observe two bands of energy levels. In the ground state the spin singlets correspond to even angular momenta and the spin triplets to odd angular momenta. Opposite correspondence is found in the excited energy band. The two bands approach each other near $B=0.5 \mathrm{~T}$, but never cross. The relation between the ground-state spin and the even/odd parity of the angular momentum remains unchanged [cf. singlets and triplets of $L=24$ marked in orange in the right upper part of Fig. 5(c)].

The distribution of the charge between the rings in the three-electron system is qualitatively similar to the twoelectron case. At zero magnetic field the electrons refuse to occupy the inner ring if its radius is too small [see Fig. 6(a)]. Some electron charge is present in the internal ring due to inter-ring tunneling, which is lifted by the application of the external magnetic field. The ground-state angular momentum at high magnetic field tends toward the value obtained for a single, external ring [see Fig. 6(b)]. For $R_{1}=140 \mathrm{~nm}$, in the range of the magnetic field presented in Fig. 6, the inter-ring coupling is not broken [cf. Figs. 5(a) and 5(b) for $R_{1}$ $=130 \mathrm{~nm}$ and $R_{1}=140 \mathrm{~nm}$ ]. In the high magnetic field limit, when the magnetic length becomes small compared to the size of the confining nanostructures, the charge distribution in few-electron systems can be identified ${ }^{28}$ with the lowest-

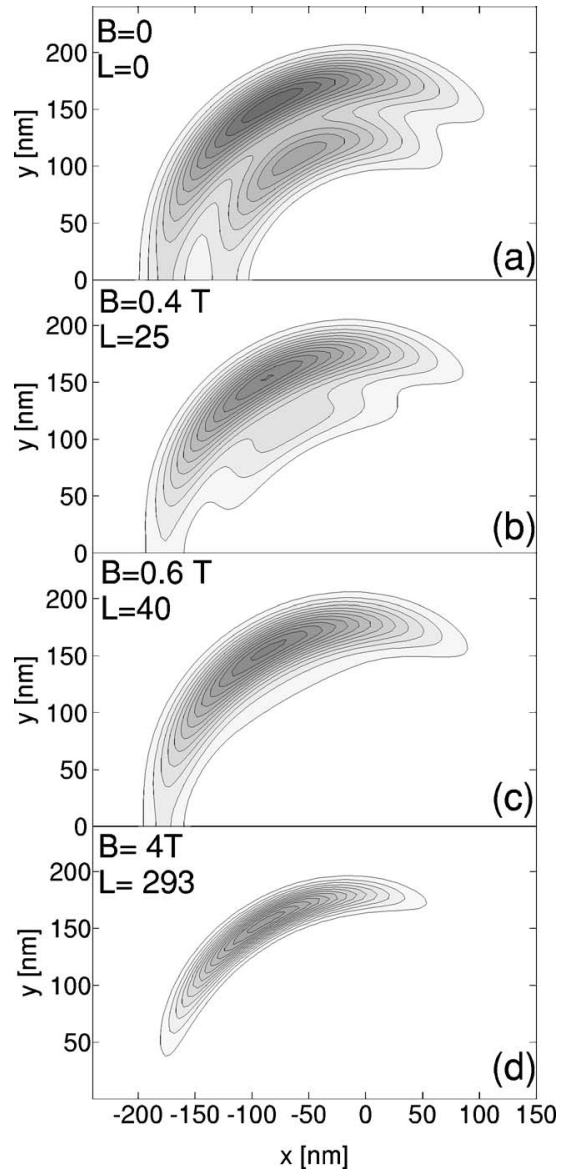

FIG. 8. Same as Fig. 7, but now for three electrons.

energy configuration of a classical system ${ }^{29}$ of point-charge particles. Therefore, one should expect that in our model, assuming equal depths of both rings, the few-electron system will eventually become entirely localized in the external ring at still higher magnetic fields.

Next, we study the evolution of the ground-state electronelectron correlations with increasing magnetic field. For this purpose we consider the pair-correlation function plots given in Figs. 7 and 8 for two- and three-electron systems, respectively. The position of one of the electrons is fixed in the middle of the external ring, namely in the point $(180 \mathrm{~nm}, 0)$. For two electrons at zero magnetic field the second electron is found with an almost equal probability in the outer and inner rings opposite to the fixed electron [Fig. 7(a)]. For 0.6 $\mathrm{T}$ [Fig. 7(d)] the second electron occupies mainly the external ring with a small leakage of the probability density to the internal ring [cf. also the orange line in Fig. 5(b)]. On the other hand, in the three-electron system at $B=0$ there is already a pronounced shift of the pair-correlation function to the external ring [Fig. 8(a)]. Figs. 7 and 8 show that the infinite magnetic field limit is obtained in two steps: first the charge is removed from the internal ring and then the angular correlations between the electrons start to increase. The Wigner type of localization, i.e., separation of electron charges in the internal coordinates, increases with each ground-state angular momentum transition tending to the point-charge limit. 

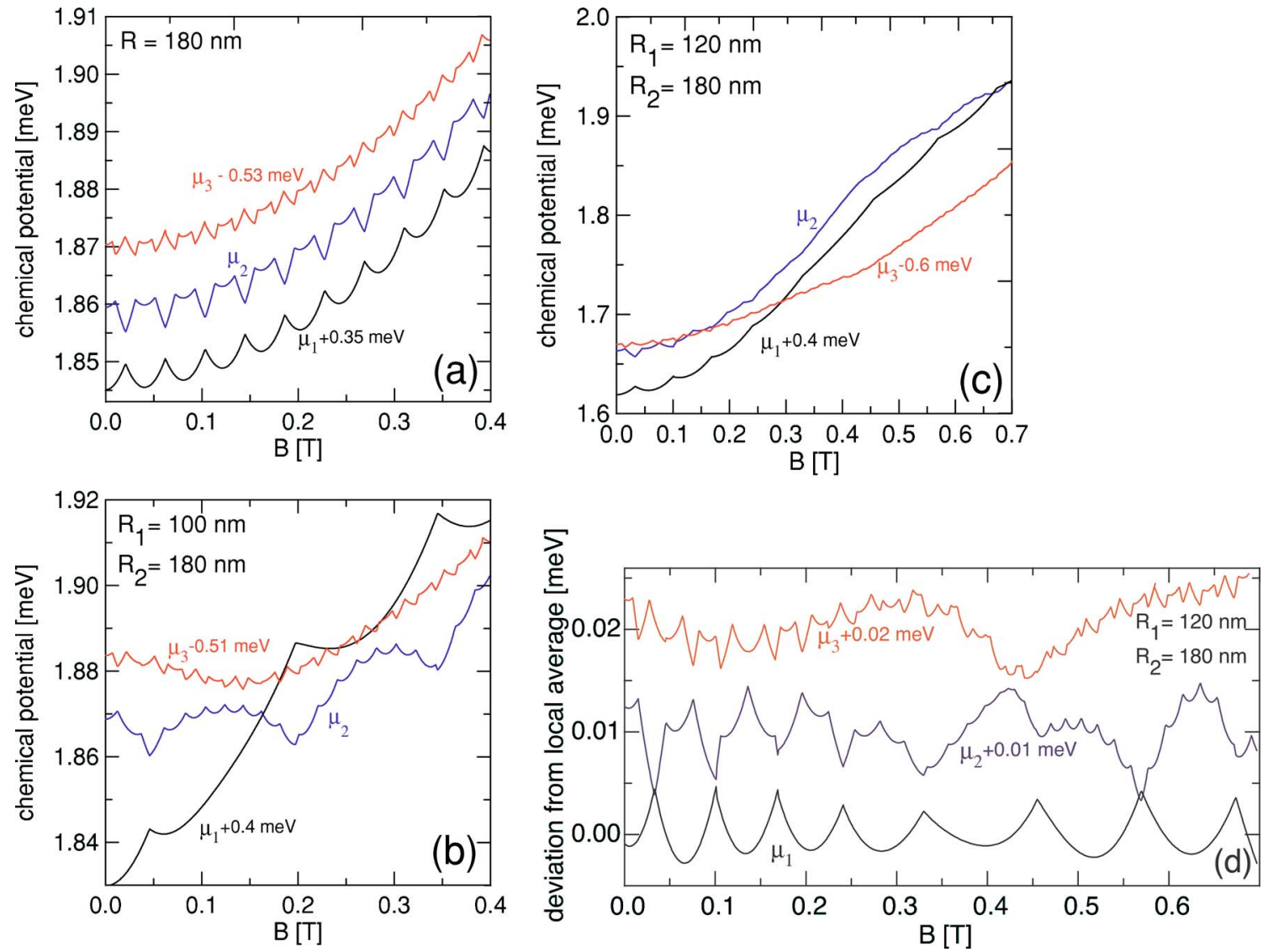

FIG. 9. (Color online) Magnetic field dependence of the chemical potentials for 1, 2, and 3 electron systems in a single ring of radius $R=180 \mathrm{~nm}$ (a), in double concentric rings of external ring radius $R_{2}=180 \mathrm{~nm}$ and internal ring radius $R_{1}=100 \mathrm{~nm}(\mathrm{~b})$, and $R_{1}=120 \mathrm{~nm}$. Chemical potentials for one and three electrons have been shifted for clarity. (d) Deviation of the chemical potentials from the local average (see text) for plot (c).

The above discussed $\mathrm{AB}$ oscillations associated with the angular momentum transitions can be measured through the magnetic field dependence of the conductance ${ }^{11}$ as performed in phase-sensitive transport spectroscopy. Such transport measurements require contacts to be attached to the nanostructure. Connection of terminals to rings formed by the surface oxidation technique $e^{4,11}$ is straightforward. On the other hand, attachment of electrodes to self-assembled rings ${ }^{14,23}$ has not been reported so far. However, the groundstate angular momentum transitions can still be extracted from the chemical potential as measured in a capacitance experiment. ${ }^{23}$ The magnetic field dependence of the chemical potentials $\mu_{N}$, defined as the ground-state energy difference of $N$ and $N-1$ electrons, is presented in Fig. 9. Figure 9(a) shows the chemical potential for a single quantum ring of radius $180 \mathrm{~nm}$. For a single electron the chemical potential is equal to the ground-state energy. The potential exhibits cusps having a $\Lambda$ shape at the angular momentum transitions. These $\Lambda$ cusps are translated into $V$-shaped cusps of the chemical potential for the two-electron system. The angular momentum transitions in the two-electron system are twice as frequent ${ }^{10}$ as for $N=1$, hence in the $\mu_{2}$ plot we observe two $\Lambda$ cusps per one V cusp. Similarly, in the cusps' pattern of the three-electron chemical potential we obtain three $\Lambda$ 's per two V's. Below $0.7 \mathrm{~T}$ for the double-ring structure with $R_{1}=140$ and $R_{2}=180 \mathrm{~nm}$, we obtain qualitatively the same spectrum of a single-ring type, only the $\mathrm{AB}$ oscillations period is increased due to the reduced effective $R$ value. This is because for $R_{1}=140 \mathrm{~nm}$ the inter-ring coupling is not broken by the magnetic field for $B<0.7 \mathrm{~T}$ [see Figs. 4, 5(a), and $6(\mathrm{a})]$. The occupied orbitals are equally distributed between the rings.

Figure 9(b), for the doubled ring with internal radius $R_{1}$ $=100 \mathrm{~nm}$, corresponds to the situation when a small magnetic field localizes the single-electron ground states in the internal ring and ejects the entire charge of the two- and three- electron systems to the external ring [see Figs. 5(b) and 6(a)]. As a consequence, for $\mu_{2}$ we observe seven to eight $\Lambda$ cusps between each couple of V's. On the other hand, the pattern of cusps in the chemical potential of the three-electron system resembles the single-ring case [Fig. 9(a)], only below $B<0.1 \mathrm{~T}$ a small perturbation of the pattern is observed.

Figure 9(c) shows the chemical potentials for $R_{1}$ $=120 \mathrm{~nm}$, for which the inter-ring tunnel coupling is quite significant at $B=0$, but becomes suppressed in the studied range of magnetic field [see Figs. 4, 5(a), 5(b), and 6] for all considered $N$. Note that for $N=1$ and 2, the range of the chemical potential modification by the magnetic field is an order of magnitude larger than for a single ring [see Fig. 9(a)]. A distinctly larger range of chemical potential variation can also be noticed for $N=1$ in Fig. 9(b). This increase is due 
to the magnetic field lifting of the inter-ring coupling present at $B=0$. For larger $N$ the Coulomb repulsion weakens the tunnel coupling at $B=0$, which explains the weaker dependence of the envelopes of $\mu_{3}$ and $\mu_{2}$ in Fig. 9(b) and $\mu_{3}$ in Fig. 9(c).

In order to extract the fine features of the cusps' pattern we fitted slowly varying sixth order polynomials to the chemical potentials in Fig. 9(c) and then subtracted from $\mu_{N}$ this local average provided by the fitted polynomial. The result is displayed in Fig. 9(d). For $N=1$ we see an enlargement of the $\mathrm{AB}$ oscillation period as the electron becomes localized in the inner ring. The low magnetic field $\Lambda-\mathrm{V}$ cusp sequences for $N=2$ and 3 resemble the single-ring localization [see Fig. 9(a)]. For $B>0.45 \mathrm{~T}$ when both electrons are ejected to the external ring and the single electron is localized in the inner ring, we see in $\mu_{2}$ several $\Lambda$ 's per one $\mathrm{V}$, as in Fig. 9(b). For $\mu_{3}$ the single-ring type of pattern is found above $B>0.45 \mathrm{~T}$. In the transition region $(0.35 \mathrm{~T}<B$ $<0.45 \mathrm{~T})$ the cusp structure is less pronounced. This is due to the fact that in the $B$ range corresponding to the transition of the electrons to the external ring, the angular momentum increases very fast, tending toward the angular momentum of the ground state in the single quantum ring [see Figs. 5(a), 5(c), and 6] of radius $R=180 \mathrm{~nm}$.

\section{SUMMARY AND CONCLUSIONS}

We studied the coupling between concentric rings for the few-electron eigenstates using the exact diagonalization approach. We find that the strength of the tunnel coupling decreases with angular momentum since the centrifugal potential favors the localization of the electrons in the external ring. At high magnetic field, for which the ground state corresponds to high angular momentum, the tunnel coupling between the rings is suppressed and the energy spectrum becomes decoupled into spectra of separate external and internal rings. The ground state for the single electron becomes entirely localized in the inner ring due to the diamagnetic term of the Hamiltonian, enhancing the localization of the electron orbits. In contrast, the few-electron states at high magnetic field become localized in the external ring to minimize their mutual Coulomb repulsion. In our model, assuming a similar radial confinement potential near the centers of both rings, we find that the order of the spin-orbital groundstate symmetries is not perturbed by the inter-ring coupling, only the stability intervals of the subsequent ground states are affected by the coupling. The modification of the electron distribution between the external and internal rings is translated into the frequency of the ground-state angular momentum transitions on the magnetic field scale. The electron distribution can be extracted from the cusp patterns of the single-electron charging lines, i.e., the chemical potential dependence on the magnetic field. Suppression of the tunnel inter-ring coupling and localization of the ground states in one of the rings under the influence of a magnetic field is accompanied by a distinctly stronger increase of the chemical potentials compared to the charging spectra in which the charge distribution between the rings is not modified.

\section{ACKNOWLEDGMENTS}

This work was supported by the Flemish Science Foundation (FWO-Vl) and the Belgian Science Policy and the EU-network of excellence SANDiE. B.S was supported by the EC Marie Curie IEF Project No. MEIF-CT-2004-500157.
*Electronic address: Francois.Peeters@ua.ac.be

${ }^{1}$ Y. Aharonov and D. Bohm, Phys. Rev. 115, 485 (1959).

${ }^{2}$ R. A. Webb, S. Washburn, C. P. Umbach, and R. B. Laibowitz, Phys. Rev. Lett. 54, 2696 (1985).

${ }^{3}$ G. Timp, A. M. Chang, J. E. Cunningham, T. Y. Chang, P. Mankiewich, R. Behringer, and R. E. Howard, Phys. Rev. Lett. 58, 2814 (1987).

${ }^{4}$ A. Fuhrer, S. Lüscher, T. Ihn, T. Heinzel, K. Ensslin, W. Wegscheider, and M. Bichler, Nature (London) 413, 822 (2001).

${ }^{5}$ W. G. van der Wiel, Yu. V. Nazarov, S. De Franceschi, T. Fujisawa, J. M. Elzerman, E. W. G. M. Huizeling, S. Tarucha, and L. P. Kouwenhoven, Phys. Rev. B 67, 033307 (2003).

${ }^{6}$ S. Pedersen, A. E. Hansen, A. Kristensen, C. B. Sorensen, and P. E. Lindelof, Phys. Rev. B 61, 5457 (2000).

${ }^{7}$ M. Büttiker, Y. Imry, R. Landauer, and S. Pinhas, Phys. Rev. B 31, 6207 (1985).

${ }^{8}$ S. Viefers, P. Koskinen, P. Singa Deo, and M. Manninen, Physica E (Amsterdam) 21, 1 (2004).

${ }^{9}$ T. Chakraborty and P. Pietiläinen, Phys. Rev. B 50, 8460 (1994).

${ }^{10}$ K. Niemielä, P. Pietiläinen, P. Hyvönen, and T. Chakraborty, Europhys. Lett. 36, 533 (1996).

${ }^{11}$ U. F. Keyser, C. Fühner, S. Borck, R. J. Haug, M. Bichler, G. Abstreiter, and W. Wegscheider, Phys. Rev. Lett. 90, 196601
(2003).

${ }^{12}$ A. Emperador, F. Pederiva, and E. Lipparini, Phys. Rev. B 68, 115312 (2003).

${ }^{13}$ J. I. Climente, J. Planelles, and F. Rajadell, J. Phys.: Condens. Matter 17, 1573 (2005).

${ }^{14}$ T. Mano, T. Kuroda, S. Sanguinetti, T. Ochiai, T. Tateno, J. Kim, T. Noda, M. Kawabe, K. Sakoda, G. Kido, and N. Koguchi, Nano Lett. 5, 425 (2005).

${ }^{15}$ C. Yannouleas and U. Landman, Phys. Rev. Lett. 82, 5325 (1999).

${ }^{16}$ S. Nagaraja, J.-P. Leburton, and R. M. Martin, Phys. Rev. B 60, 8759 (1999).

${ }^{17}$ A. Harju, S. Siljamäki, and R. M. Nieminen, Phys. Rev. Lett. 88, 226804 (2002).

${ }^{18}$ M. Marlo, A. Harju, and R. M. Nieminen, Phys. Rev. Lett. 91, 187401 (2003).

${ }^{19}$ A. Wensauer, O. Steffens, M. Suhrke, and U. Rössler, Phys. Rev. B 62, 2605 (2000).

${ }^{20}$ J. J. Palacios and P. Hawrylak, Phys. Rev. B 51, 1769 (1995).

${ }^{21}$ M. Pi, A. Emperador, M. Barranco, F. Garcias, K. Muraki, S. Tarucha, and D. G. Austing, Phys. Rev. Lett. 87, 066801 (2001).

${ }^{22}$ B. Partoens and F. M. Peeters, Phys. Rev. Lett. 84, 4433 (2000); Europhys. Lett. 56, 86 (2001). 
${ }^{23}$ A. Lorke, R. J. Luyken, A. O. Govorov, J. P. Kotthaus, J. M. Garcia, and P. M. Petrof., Phys. Rev. Lett. 84, 2223 (2000).

${ }^{24}$ B. Szafran, F. M. Peeters, and S. Bednarek, Phys. Rev. B 70, 125310 (2004).

${ }^{25}$ B. J. Baelus, S. V. Yampolskii, and F. M. Peeters, Phys. Rev. B 66, 024517 (2002).

${ }^{26}$ S. M. Reimann and M. Manninen, Rev. Mod. Phys. 74, 1283
(2002).

${ }^{27}$ M. B. Tavernier, E. Anisimovas, F. M. Peeters, B. Szafran, J. Adamowski, and S. Bednarek, Phys. Rev. B 68, 205305 (2003).

${ }^{28}$ B. Szafran, F. M. Peeters, S. Bednarek, and J. Adamowski, Phys. Rev. B 69, 125344 (2004).

${ }^{29}$ V. M. Bedanov and F. M. Peeters, Phys. Rev. B 49, 2667 (1994). 